\title{
Satisfacción Laboral en los Entornos de Trabajo. Una exploración cualitativa para su estudio
}

\author{
Job satisfaction in work settings. A qualitative approach for study. \\ Sánchez Trujillo Magda Gabriela, Ma. De Lourdes E. García Vargas \\ Escuela Superior Tepeji, Universidad Autónoma del Estado de Hidalgo, Tepeji del Rio, Hidalgo, México. \\ Correo-e: mgabyst@gmail.com
}

\begin{abstract}
Resumen-El éxito de las organizaciones modernas radica de una manera importarte en la capacidad de integrar a los trabajadores en los proyectos empresariales de las compañías, de tal forma que los empleados consideren los objetivos de la organización como propios. Considerando el factor humano como la base para lograr la competitividad, es importante contar con el personal más calificado y motivado posible. Partiendo de este hecho, éste factor se ha convertido en un recurso estratégico que se debe optimizar. El presente trabajo distingue la relación entre condiciones de trabajo y la satisfacción laboral entre los trabajadores que laboran en empresas del mismo giro ubicadas en diferentes entidades de México, a través de un enfoque cualitativo que se llevó a cabo guiado de un cuestionario semi-estructurado. Los resultados muestran variaciones, los cuales se relacionan con las condiciones de trabajo, comunicación entre empleado-jefe, logro y motivación.
\end{abstract}

Palabras clave - Condición de trabajo, relación laboral, satisfacción laboral.

Abstract-The success of organizations lies in optimal management of the human resource. The human factor is the basis of competitiveness, therefore the importance of having the most qualified and motivated as possible. The present work seeks to identify the relationship between working conditions and job satisfaction in the same business companies located in different states of Mexico, through a qualitative study it was conducted by applying a semi-structured questionnaire. The results showed variations which are related to working conditions, employeemanager communication, achievement and motivation.

Key Word - Job satisfaction, labor relations, working conditions.

\section{INTRODUCCIÓN}

Para que una empresa contribuya con eficiencia a sus obligaciones con los empleados, accionistas y la sociedad, sus directivos deben generar una relación entre la organización y sus trabajadores que satisfaga las necesidades siempre cambiantes de ambas partes. Como mínimo la organización espera que los empleados desempeñen de manera confiable las tareas que se les asignen y con los estándares que se les fijen, que sigan las normas establecidas para administrar el sitio de trabajo. Con frecuencia, la dirección espera más: que tomen iniciativas, que se supervisen por sí mismos, que continúen desarrollando nuevas habilidades y que se respondan a las necesidades comerciales. Por su parte los empleados también esperan como mínimo que la organización les proporcione una paga equitativa, condiciones seguras de trabajo y un trato justo. Igual que la dirección, los empleados también esperan más, dependiendo de la magnitud de sus necesidades de seguridad, situación laboral y económica, entorno, retos, oportunidades, poder y responsabilidad. De esta forma la pretensión de las expectativas que tenga cada una de las partes variará de organización a organización y de persona a persona, el reto es alinear los objetivos e intereses organizacionales con los particulares.

Por lo anterior, la satisfacción laboral es un tema de interés destacado en el ámbito del trabajo, dado que han sido muchos los estudios que se han realizado al respecto, con la intensión de determinar cuáles son los múltiples factores que influyen en el bienestar de las personas en sus ambientes laborales, así como determinar cómo influye la satisfacción en el desempeño de los individuos en sus puestos de trabajo. De acuerdo con [1], la satisfacción laboral produce importantes aspectos vinculados con los empleados y las organizaciones, desde el desempeño laboral hasta la salud y la calidad de vida.

Lo anterior expresa que si el propósito de la organización es crecer estratégicamente, corresponde a la empresa conocer a las personas que laboran dentro de ella, lo que implica valorar las condiciones de trabajo y la satisfacción laboral. 
Para ello es importante saber el punto de vista del trabajador y el grado de satisfacción laboral que experimenta. En este sentido se plantea la interrogante ¿qué impacto produce en la organización la satisfacción laboral de los trabajadores?

Como eje de análisis en este estudio se consideró el diseño fenomenológico que se enfoca en experiencias individuales subjetivas de los participantes ya que se pretende reconocer las percepciones de las personas y el significado de un fenómeno concreto que es la satisfacción laboral. Se trata por tanto de un estudio cualitativo con alcance exploratorio, descriptivo, fenomenológico.

Esta publicación se considera oportuna tanto para el sector empresarial como para el estudio de la administración ya que muestra la perspectiva del empleado hacia las condiciones de trabajo propias de la organización, medida en grados de satisfacción laboral, lo cual permite en palabras de [2] identificar las áreas de oportunidad y de desarrollo potencial para la mejora de los procesos que conduzcan a obtener mejores beneficios económicos y calidad. La investigación puede tomarse como una guía respecto a las variables a considerar en futuros estudios. De esta manera el trabajo plantea como objetivo realizar un estudio para distinguir el grado en que las condiciones de trabajo se relacionan con la satisfacción laboral, a través de un enfoque cualitativo que permita analizar la percepción y expectativas de los trabajadores respecto a su ámbito laboral.

\section{CONTENIDO}

\section{A. Revisión de literatura}

En los últimos 30 años se han incrementado los a trabajadores donde se les ha cuestionado sobre qué factores del trabajo son más importantes en términos de satisfacción o insatisfacción en el trabajo. Estos estudios son de interés particular al determinar qué satisfactores (objetivos o resultados) busca el trabajador en el medio ambiente organizacional y qué insatisfactores es probable que resulten en la conducta defensiva.

En la actualidad, no existe una definición unánime respecto del concepto de satisfacción laboral. Los autores [3], exponen que a pesar de su larga tradición en la piscología de las organizaciones, el concepto de satisfacción laboral ha sido definido de múltiples formas, tantas como autores han teorizado sobre él. Para estos autores, existen una serie de definiciones que hace referencia a la satisfacción laboral como un estado emocional, sentimientos o respuestas objetivas.

\section{Satisfacción Laboral}

Para [4], la satisfacción de un trabajador con su trabajo y su entorno laboral, es un aspecto sumamente relevante para los encargados de dirigir una empresa. Son numerosas las investigaciones que han constatado que la fidelidad de un trabajador con su empresa, su productividad, su nivel de ausentismo o su rotación laboral dependen en un alto nivel de la satisfacción laboral.

La satisfacción laboral se puede relacionar directamente con el conocimiento de la persona dentro de una organización. Esta experiencia se transforma en la percepción del trabajador, y esta última culmina en un componente emocional. Todos estos factores desembocan en la manera de actuar del individuo. [5]

Otra concepción de satisfacción laboral nos la da [6], donde la conceptualiza como una actitud o conjunto de actitudes desarrollados por la persona hacia su situación de trabajo, estas acciones pueden ir referidas hacia el trabajo en general o hacia ciertas facetas específicas del mismo. Por lo anterior, la satisfacción laboral es básicamente un concepto globalizador con el que se hace referencia a las actitudes de las personas en diversos aspectos de su trabajo.

Como se ha observado, la mayoría de las definiciones coinciden que la satisfacción laboral es una percepción que da como resultado un estado emocional del trabajador. Pero aquí cabe la pregunta ¿qué elementos o factores influyen es esa percepción? Al respecto [7], exponen que la satisfacción laboral puede ser percibida a través de algunos elementos, como, por ejemplo: que el personal sea bien recompensado a través de sueldos y salarios acordes, obviamente con las expectativas de cada uno; que las condiciones de trabajo sean adecuadas, no peligrosas o incomodas, lo cual mejora su desempeño; que el personal busque que su jefe inmediato sea amigable y compresible y que los escuche cuando sea necesario.

\section{La Satisfacción: Un factor no causal}

Según [8] la relación satisfacción - desempeño puede ser inversa: el nivel de desempeño puede ser un factor causal en la satisfacción en el trabajo, siendo las recompensas intrínsecas y extrínsecas variables de refuerzo. Las variables intrínsecas son recompensas como "el sentimiento de haber realizado algo útil". Las recompensas extrínsecas son paga, promoción, posición, etc. Como se muestra en la figura 1.

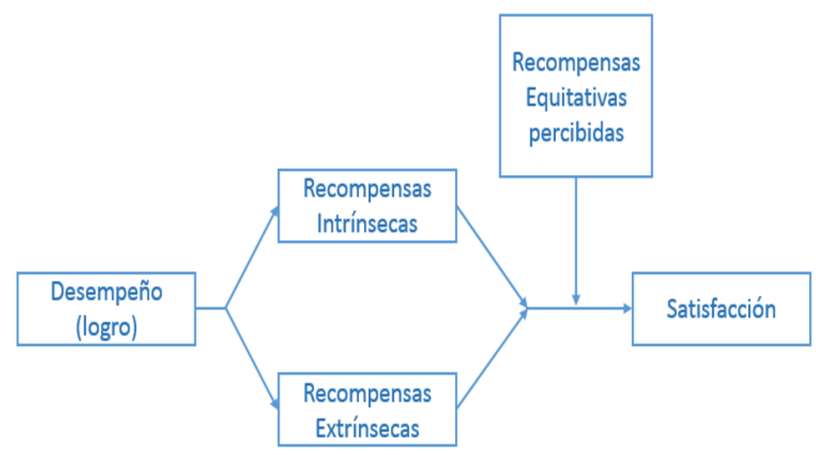

Fig. 1. Modelo de desempeño-recompensa-satisfacción. 
El desempeño pude conducir a recompensas que a su vez producen satisfactores. La percepción de equidad se muestra también como una variable importante, ya que en cierta medida la satisfacción puede ser una función del nivel de recompensas en relación con las recompensas dadas a otros.

Existe evidencia de estudios que han medido la satisfacción laboral, la cual se evalúa casi siempre sondeando a las personas en base a percepciones hacia sus empleos, ya sea mediante cuestionarios o entrevistas [1]; entre ellos se encuentra, por ejemplo, [9] sobre el grado de satisfacción laboral en instituciones de enfermería. En él, los autores integraron las siguientes variables en su instrumento: carga de trabajo, demanda física, salario promedio, horas de trabajo, programas de carrera, valoración de las habilidades y la experiencia, visión social de la profesión, nivel de estrés, trabajo en equipo y apoyo de los compañeros, seguridad del área de trabajo, fomento a la autonomía y equipamiento del área de trabajo.

\section{Condiciones de Trabajo}

La actividad laboral está sujeta a variables muy diversas y el conjunto de todas ellas forman las condiciones de trabajo. Por condiciones de trabajo se entiende el estado del ambiente de trabajo relacionado con la seguridad e higiene [10]. En este sentido, en los últimos 20 años se han incorporado nuevos parámetros, las denominadas políticas de riesgos laborales, es decir, normas y pautas que deben regir una actividad para que ésta tenga las suficientes garantías de seguridad física para el trabajador, así como para su salud.

Además del salario, la seguridad y la salud, hay otras condiciones que igualmente influyen en la manera de realizar un trabajo, el horario es una de ellas. Existen varias modalidades, pues la empresa necesita cubrir un horario productivo y, paralelamente, los trabajadores tienen que compatibilizar su actividad y su tiempo libre. La política de incentivos es, sin duda, otro aspecto de la totalidad de las condiciones laborales, y no se refiere exclusivamente a la remuneración, sino que toma en cuenta la promoción interna de los empleados.

En relación a las condiciones de trabajo éstas se encuentran plasmadas de manera legal en la ley laboral, lo mismo que su definición en su artículo 20 [11]

Artículo 20.- Se entiende por relación de trabajo, cualquiera que sea el acto, que le de origen a la prestación de un trabajo personal subordinado a una persona, mediante el pago de un salario. [11].

La misma ley laboral en su artículo 391, explicita cuales son los componentes del contrato por la prestación de un trabajo personal que se detallan a continuación.

I. Nombres y domicilios de los contratantes.

II. Las empresas y establecimientos que abarquen.
III. Su duración o la expresión de ser por tiempo indeterminado o para obra determinada.

IV. Las jornadas de trabajo.

V. Los días de descanso y vacaciones.

VI. El monto de los salarios.

VII. Las clausulas relativas a la capacitación o adiestramiento de los trabajadores en la empresa o establecimientos que comprenda.

VIII. Disposiciones sobre la capacitación o adiestramiento inicial que se deba impartir a quienes vayan a ingresar a laboral a la empresa o establecimiento.

IX. Las bases sobre la integración o funcionamiento de las comisiones que deban de integrarse conforme a esta ley.

X. Las demás estipulaciones que convengan las partes.

Otro propósito de las condiciones de trabajo es proteger a los empleados y sirven como dispositivos de control, ya que la administración las utiliza como estándares contra los cuales aplicar sanciones o bien otorgar beneficios, con el objetivo de asegurar un nivel razonable de conformidad.

\section{METODOLOGÍA.}

De acuerdo a [12], el diseño se refiere al marco interpretativo que se utiliza en la investigación. En el caso que nos ocupa se consideró como base el diseño fenomenológico que se enfoca en experiencias individuales subjetivas de los participantes [13] ya que se pretende reconocer las percepciones de las personas y el significado de un fenómeno concreto. Se trata por tanto de un estudio cualitativo con alcance exploratorio, descriptivo, fenomenológico.

Los criterios de inclusión y exclusión para seleccionar a los participantes fueron: antigüedad, al menos 2 años en el puesto de nivel medio, buen desempeño y experiencia, además que ocuparan un puesto similar. La muestra es de dos empresas dedicadas a la manufactura y venta de prendas de vestir, la diferencia es que están ubicadas en contextos diferentes una empresa está ubicada en el estado de Hidalgo y otra en el Estado de México.

El instrumento guía integra 70 items que consideran diversas dimensiones encontradas en la revisión de la literatura tales como: condiciones de trabajo, seguridad en el trabajo, comunicación, relaciones interpersonales, clima organizacional y motivación. Para el análisis de los resultados se agruparon los en los siguientes parámetros:

\section{Satisfactorio}

No satisfactorio
Aquellas respuestas que incluyeran términos tales como "me interesa", "de gusta", "efectivo", etcétera, Aquellas en las que se hiciera alusión a términos acuerdo", "está bien", "me 
relacionados con una calificación negativa: "en desacuerdo", "está mal", "es bajo", "negativo", "escaso", entre otras.

La definición de las categorías queda como sigue:

Seguridad en el trabajo. Aspectos relacionados directamente con los derechos del trabajador y con condiciones laborales dignas.

Condiciones de trabajo. Las características siguientes: nivel de ingreso, jornada laboral, descansos , prestaciones (vacaciones, aguinaldo, utilidades, transporte, servicios médicos), capacitación para el trabajo, oportunidades de desarrollo profesional (ascensos o continuar estudios), seguridad e higiene (material y equipo requerido, limpieza y seguridad en el área de trabajo, instalaciones sanitarias, suministro de agua potable, lugar de descanso, comedor), incentivos (en efectivo o de reconocimiento) y carga de trabajo.

Relaciones interpersonales. La forma en que se asocia con más personas dentro de la organización.

La Comunicación. Refiere la manera, acción y resultado de comunicarse con el jefe, subordinados y compañeros.

Motivación. Los estímulos que mueven a la persona a realizar determinadas acciones y persistir en ellas logro y afiliación.

Clima organizacional. Los factores del sistema organizacional y las tendencias motivacionales que se

\begin{tabular}{|c|c|}
\hline \multicolumn{2}{|c|}{ Hidalgo } \\
\hline No Satisfactorio & Satisfactorio \\
\hline \multicolumn{2}{|c|}{ Seguridad en el Trabajo } \\
\hline Seguridad & \\
\hline \multicolumn{2}{|c|}{ Condiciones de trabajo } \\
\hline \multicolumn{2}{|l|}{ Ingreso } \\
\hline \multicolumn{2}{|l|}{$\begin{array}{l}\text { Jornada de trabajo } \\
\text { Prestaciones } \\
\text { Capacitación }\end{array}$} \\
\hline \multicolumn{2}{|l|}{ Desarrollo } \\
\hline \multicolumn{2}{|l|}{ Seguridad e Higiene } \\
\hline \multicolumn{2}{|l|}{ Incentivos } \\
\hline \multicolumn{2}{|l|}{ Carga de trabajo } \\
\hline \multicolumn{2}{|c|}{ Relaciones Interpersonales } \\
\hline \multicolumn{2}{|l|}{$\begin{array}{l}\text { Jefes } \\
\text { Compañeros } \\
\text { Subordinados }\end{array}$} \\
\hline \multicolumn{2}{|c|}{ Comunicación } \\
\hline \multicolumn{2}{|l|}{$\begin{array}{l}\text { Jefes } \\
\text { Compañeros }\end{array}$} \\
\hline \multicolumn{2}{|l|}{ Subordinados } \\
\hline \multicolumn{2}{|c|}{ Motivación } \\
\hline \multicolumn{2}{|l|}{ Intrínseca } \\
\hline \multicolumn{2}{|l|}{ Logro } \\
\hline \multicolumn{2}{|l|}{$\begin{array}{l}\text { Afiliación } \\
\text { Extrínseca }\end{array}$} \\
\hline \multicolumn{2}{|c|}{ Clima Organizacional } \\
\hline Clima & \\
\hline
\end{tabular}

traducen en un comportamiento que tiene consecuencias sobre la organización (productividad, satisfacción, rotación, etc.).

La forma de interpretar los resultados está en función de las respuestas: $\mathrm{Si}$ son favorables esto muestra un grado de satisfacción suficiente y, por el contrario, las desfavorables implican un grado deficiente de satisfacción.

\section{RESULTADOS}

Tabla 2. Resultados de Satisfacción Laboral en las empresas En la tabla 2 se presentan los resultados encontrados referentes a las respuestas satisfactorias y no satisfactorias de satisfacción laboral de las empresas estudiadas.

\section{Seguridad en el trabajo}

En la tabla se observa que en la dimensión Seguridad en el trabajo existe similitud entre ambas empresas. La persona entrevistada en la empresa hidalguense considera que su desempeño es bueno y no tiene temor de perder tu trabajo.

La persona en la empresa mexiquense expreso que no hay inseguridad en el trabajo de momento.

\section{Condiciones de Trabajo}

Respecto al ingreso el trabajador en estado de México considera que es adecuado para cubrir sus condiciones de vida. Sin embargo el trabajador hidalguense considera que el sueldo cubre sus necesidades básicas pero que si le gustaría mejorar

\begin{tabular}{|c|c|}
\hline \multicolumn{2}{|c|}{ Estado de México } \\
\hline No Satisfactorio & Satisfactorio \\
\hline \multicolumn{2}{|c|}{ Seguridad en el Trabajo } \\
\hline Seguridad & \\
\hline \multicolumn{2}{|c|}{ Condiciones de trabajo } \\
\hline \multicolumn{2}{|l|}{$\begin{array}{l}\text { Ingreso } \\
\text { Jornada de trabajo } \\
\text { Prestaciones } \\
\text { Capacitación }\end{array}$} \\
\hline \multicolumn{2}{|l|}{ Desarrollo } \\
\hline \multicolumn{2}{|l|}{$\begin{array}{l}\text { Seguridad e Higiene } \\
\text { Incentivos }\end{array}$} \\
\hline \multicolumn{2}{|l|}{ Carga de trabajo } \\
\hline \multicolumn{2}{|c|}{ Relaciones Interpersonales } \\
\hline \multicolumn{2}{|l|}{$\begin{array}{l}\text { Jefes } \\
\text { Compañeros }\end{array}$} \\
\hline \multicolumn{2}{|l|}{ Subordinados } \\
\hline \multicolumn{2}{|c|}{ Comunicación } \\
\hline \multicolumn{2}{|l|}{$\begin{array}{l}\text { Jefes } \\
\text { Compañeros } \\
\text { Subordinados }\end{array}$} \\
\hline \multicolumn{2}{|c|}{ Motivación } \\
\hline \multicolumn{2}{|l|}{ Intrínseca } \\
\hline \multicolumn{2}{|l|}{$\begin{array}{l}\text { Logro } \\
\text { Afiliación }\end{array}$} \\
\hline \multicolumn{2}{|l|}{ Extrinseca } \\
\hline \multicolumn{2}{|c|}{ Clima Organizacional } \\
\hline Clima & \\
\hline
\end{tabular}


sus condiciones. En ambos casos el pago es por tarjeta electrónica y les parece cómoda la forma de pago.

En cuanto a la jornada de trabajo en ambos casos manifestaron agrado ante el número de horas de trabajo, así como el tiempo de receso en planta y el día de descanso.

Referente a prestaciones no manifestaron problemas respecto a las prestaciones de ley (seguridad social, aguinaldo, vacaciones, en el caso del entrevistado del estado de Hidalgo le gustaría que la empresa tuviera un espacio más confortable para el comedor. El empleado de la empresa mexiquense se mostró conforme con el comedor y solo mencionó que si le gustaría les ofrecieran servicio de transporte por seguridad y economía.

La capacitación es un aspecto satisfactorio en ambas empresas, los trabajadores manifestaron que la capacitación les ayuda a hacer más eficiente su trabajo y en el caso del empleado hidalguense mencionó que le ha tocado reproducir algunos cursos a sus subordinados lo que le hace sentir satisfecho en su empleo.

El tema de Desarrollo resulto insatisfactorio en ambos trabajadores, en la empresa hidalguense no hay más puestos que ofrecer menciona el entrevistado y en el caso de la empresa mexiquense el entrevistado expresó que ha solicitado tiempo para continuar estudiando, le dieron oportunidad pero hubo cambio de jefe de área y ya no le brindan el apoyo, lo cual considera un impedimento para subir de puesto dentro de la empresa o conseguir otro mejor puesto fuera de la empresa.

Seguridad e Higiene es un aspecto satisfactorio en ambas empresas los trabajadores mencionaron que se les proporciona equipo de seguridad y protección en su lugar de trabajo, están conformes con las instalaciones y la higiene del lugar.

Los Incentivos en la empresa mexiquense consisten en bonos por puntualidad, reconocimientos por productividad y recientemente se implementó un programa de apoyo a útiles escolares y lentes para los empleados. El trabajador en la empresa hidalguense se mostró indiferente en este rubro ya que dijo los incentivos son esporádicos no hay ningún tipo de programa o sistema por lo que no es algo que se tenga seguridad en ello. Se observa que los incentivos si pueden afectar la satisfacción en el empleado.

Analizando el aspecto de carga de trabajo el trabajador del estado de México no está satisfecho con la carga de trabajo diaria considera que tiene mayor responsabilidad de lo que su puesto implica y no tiene tiempo suficiente para desempeñar sus labores. El trabajador de la empresa en Hidalgo se mostró conforme con su carga y responsabilidad de trabajo.

\section{Relaciones Interpersonales}

Se encuentra que las relaciones personales son satisfactorias en el empleado de la empresa en Hidalgo su relación con jefes, compañeros y subordinados considero que es buena. En el caso del empleado de la empresa en estado de México considera que su relación con jefes y compañeros es satisfactoria, pero que con su subordinado ha tenido ciertos conflictos, sin embargo dijo buscar maneras de solucionarlas.

\section{Comunicación}

Los resultados de comunicación con jefes y compañeros en ambas empresas es satisfactoria, lo que resulta diferente es el aspecto de comunicación con subordinados en la empresa en Hidalgo, el entrevistado dijo que solo la necesaria que ésta se limita a cuestiones de trabajo.

\section{Clima Organizacional}

Se observa que en la empresa hidalguense el clima organizacional es satisfactorio en contraste con la empresa mexiquense donde el empleado se refirió a un ambiente pobre, las políticas de la empresa plasman una cosa pero en la práctica no todos están integrados y se pueden observar grupos

\section{Motivación}

Respecto de la motivación intrínseca en ambas empresas es nulo el fomento de aspiración a nuevos puestos o proyectos que impliquen retos para los trabajadores. En tanto que en la empresa hidalguense se preocupan por integrar el trabajo en equipo de sus empleados, en la mexiquense no se preocupan por este aspecto mencionó el trabajador aunque las mismas labores propician que se trabaje de esta manera. La motivación extrínseca se da en ambas empresas cuando se recompensa por realizar trabajo extraordinario.

\section{CONCLUSIONES}

No es fácil decir simplemente que las empresas estudiadas mantienen condiciones de trabajo que propician satisfacción laboral, al plantear el objetivo de realizar un estudio para distinguir el grado en que las condiciones de trabajo se relacionan con la satisfacción laboral, se ha podido determinar la percepción y expectativas de los trabajadores respecto a su ámbito laboral. Queda claro que la pretensión de las expectativas que tenga cada una de las partes, es decir empresa - empleado variará de organización a organización y de persona a persona.

Lo que se alcanza a apreciar es que hay claras diferencias en la manera de percibir la satisfacción laboral, pues aun cuando la empresa hidalguense muestra 15 aspectos favorables y 5 no satisfactorios y la empresa mexiquense 14 aspectos favorables contra 6 no satisfactorios éstos últimos se relacionaron con el fomento de las oportunidades de trabajo, los incentivos, la carga de trabajo, la comunicación entre empleado-jefe y las necesidades de logro y el clima laboral. 
Se observa también que la motivación extrínseca planteada por [8] si resulta ser en la investigación una variable de refuerzo de recompensa que da satisfacción laboral a los empleados de ambas empresas. Varios factores intrínsecos del trabajo, por ejemplo cuando éste trabajo representa un desafío o interés, o cuando están presentes éstos términos, son quizá factores importantes que contribuyen a la satisfacción en el trabajo. Cuando los factores intrínsecos están ausentes la insatisfacción tiende a enfocarse en factores extrínsecos, como en estos casos.

Existen quizá latentes necesidades de autorrealización y progreso personal en los trabajadores, pero una expresión considerable de tales necesidades depende de algún nivel de satisfacción respecto a requerimientos fisiológicos y necesidades de seguridad.

El salario parece ser un motivador para el empleado de una empresa y un insatisfactor para el otro. Cuando la compensación económica está de acuerdo con los logros, el avance del trabajo pareciera que actúa interdependientemente con estos factores como un motivador y satisfactor. Cuando el salario no está acorde con estos factores, sirve como un insatisfactor para buenos empleados.

La insatisfacción con el trabajo, en sí no es alta. Se puede obtener inferencia a partir de la investigación de que las personas desean trabajo más desafiante, cuando menos en la medida en que el trabajo está asociado con otros resultados deseables, tales como un mayor puesto o mejor salario.

Se considera, además de seleccionar, utilizar y desarrollar a las personas más calificadas y la tecnología más apropiada, el resto de la administración es en dos sentidos:

a) Crear un medio ambiente organizacional en el cual se minimice la conducta no productiva y costosamente defensiva y

b) Crear una organización en que las personas tengan oportunidad de expresar sus necesidades superiores por medio de logros exitosos que benefician también a la organización.

Finalmente lejos de considerar un trabajo terminado la investigación puede ser utilizada como una alternativa que guie futuros estudios. La creación de las condiciones mencionadas requiere un reexamen de muchas de las suposiciones básicas acerca de los individuos y de la administración de los mismos.

\section{AGRADECIMIENTOS}

Agradecemos ampliamente a las personas de las empresas que amablemente participaron con sus respuestas en el estudio.

\section{REFERENCIAS}

[1]. Spector, P. (2002). Psicología industrial y organizacional: investigación y práctica. México: El Manual Moderno

[2]. Galaz-Fontes, Jesús (2003). La satisfacción laboral de los académicos en una universidad estatal pública, México: ANUIES.

[3]. Chiang, V., Ojeda, H. J. (2013). Estudio de la relación entre satisfacción laboral y el desempeño de los trabajadores de las ferias libres. Contaduría y Administración, Abril-Junio, 39-60.

[4]. Rico Belda, P. (2012). Satisfacción laboral de los asalariados en España. Revista de Métodos Cuantitativos para la Economía y la Empresa, Diciembre, 137-158.

[5]. Castro, A., Contreras P., \& Montoya R. (2009). Grado de satisfacción laboral y condiciones de trabajo: una exploración cualitativa. Enseñanza e Investigación en Psicología. 14 (1). pp. 105-118.

[6]. Chiang, V., Salazar, B., Núñez, P. (2007). Clima organizacional y satisfacción laboral en un establecimiento de salud estatal: hospital tipo 1. Theoria, 61-76.

[7]. Rodríguez, D., Núñez, L., Cáceres, A. (2010). Estudio comparativo de la satisfacción laboral universitaria de Anzoátegui de la Universidad de Oriente. Investigación y Posgrado, Enero-Junio, 63-68.

[8]. Lawler, E., Porter, L. (1997). The effect of performance on job satisfaction, Industrial Relations. 7(20), 20-28

[9]. Hegney, Plank y Parker (2006). Extrinsic and intrinsic work values: Their impact on job satisfaction in nursing. Journal of Nursing Management, 14, 271-281

[10]. De la Cueva, M. (2002). El Nuevo Derecho Mexicano del Trabajo. (Vol. I). México, D.F., México: Porrua.

[11]. Ley Federal del Trabajo (1 de Abril de 1970).México, recuperado el 30 de Enero de 2015 de:http://www.stps.gob.mx/bp/micrositios/reforma_laboral/arc hivos/Noviembre.\%20Ley\%20Federal\%20del\%20Trabajo\%2 0Actualizada.pdf.

[12]. Álvarez-Gayou J, J. L. (2003). Cómo hacer investigación cualitativa. Fundamentos y metodología. México: Paidos Educador.

[13]. Bogdan, R. C y Biklen, S. K. (2003). Qualitative Research for Education: An introduction to Theories and. Methods. $\quad\left(4^{\text {th }}\right.$ Ed. $)$ New York: Pearson Education group 